\title{
Antiepileptic drugs use among patients attending epilepsy clinic at Jimma university teaching and referral hospital, southwest Ethiopia: Retrospective facility based cross-sectional descriptive study
}

\author{
Walelign Atalay ${ }^{1}$, Chernet Abebe $^{2}$ and Waju Beyene Salgedo ${ }^{3 *}$ \\ ${ }^{1}$ College of Health sciences, Jimma University, Jimma, Ethiopia. \\ ${ }^{2}$ Department of Internal Medicine, College of Health Sciences, Jimma University, Jimma, Ethiopia. \\ ${ }^{3}$ Department of Health Economics, management and policy, College of Health Sciences, Jimma, Ethiopia.
}

Accepted 2 May, 2017

\begin{abstract}
Antiepileptic drugs (AEDs) are primary therapeutic mode for epilepsy. Nevertheless, the risks of significant adverse effects and drug interaction increase when more than one drug is used. A retrospective crosssectional facility based study was carried out in Jimma university specialized teaching hospital, southwest Ethiopia, from June 25 to July 5, 2015. The sample size was determined by Joint Commission on the Accreditation of Health Care Organization (JCAHO) criteria by taking $5 \%$ of the total patient medical cards through systematic random sampling technique. Data were collected by trained data collectors using the pre-developed data collection format. It was entered onto computer and analyzed by SPSS 20.0 for window and the result was presented in tables and descriptive narrations. A total of 154 patients' treatment cards which contains AEDs were studied. $95(61.7 \%)$ of the subjects were males and majority, $74(48 \%)$, were in the age group of 5 to 18 years old. Generalized tonic-clonic seizure $(76.3 \%)$ was the most common type of epileptic seizure seen. Monotherapy (89.7\%) was most frequently used. Headache (64.4\%) was the commonest adverse effect complained by the patients. Almost eighty percent $(79.20 \%)$ of AED use was in accordance with indications while $20.8 \%$ were difficult to conclude whether it is correct or incorrect indications since the type of epilepsy is not specified. There was $17.7 \%$ under dose, $8.4 \%$ over dose and $21.1 \%$ AED use. There were potential drug-drug interactions in $9.4 \%$. The present study has revealed prudent use of AEDs pertaining to indications and contraindications. The classification of seizure, use of drugs with potential drug interaction, dose and duration experiences need urgent intervention of giving health education to patients to report to physicians on follow-up days.
\end{abstract}

Keywords: Drug use evaluation, epilepsy, antiepileptic drugs, drug utilization.

${ }^{*}$ Corresponding author. E-mail: wabeyene@yahoo.com.

Abbreviation: ADR, Adverse drug reaction; AEDs, antiepileptic drugs; CBE, community based education; JCAHO, Joint Commission on the Accreditation of Health care Organization; JUSH, Jimma University Specialized Hospital; NDP, national drug policy; PMHRs, patient medical health records; RDUR, retrospective drug utilization review; UK, United Kingdom; USA, United State of America; WHO, World Health Organization.

\section{INTRODUCTION}

Epilepsy is a chronic disorder characterized by recurrent seizures (Leppik, 1996). Epilepsy is the second most common chronic neurological condition seen by neurologists. It is estimated that there are $5,500,000$ persons with epilepsy in India, 2,000,000 in USA and 300,000 in UK (Sridharan, 2002). 
Around $90 \%$ of the people with epilepsy in developing countries are not receiving appropriate treatment due to cultural attitude, lack of prioritization, poor health system infrastructure and inadequate supply of antiepileptic drugs (AEDs) (Scott et al., 2001). Economic factors are important determinants of clinical decision making and the degree of effect depends on the country and health systems. In developing countries these may be more related to patient's ability to pay and that of availability of drugs (Wyllie, 2001).

The pharmacokinetics of the most frequently prescribed AEDs are complex, which makes dosing and monitoring difficult in any age group; however, treatment decisions become more complicated with the older patient (Joint commission on the Accreditation of Health care Organizations, 1995). Age related alterations in physiology can affect AED pharmacology and limit the choice of medications. There is a lack of safety and efficacy information to guide clinical decisions about AED therapy in older people. The complexity of medical problems and co-medications among older people requiring AEDs can result in an increased likelihood of drug-related problems and drug interactions, which in turn can affect seizure control and toxicity (Schacter et al., 1998). Enzyme-inducing drugs such as carbamazepine, oxcarbazepine, phenytoin, Phenobarbital, primidone and topiramate may accelerate the metabolism of both estrogen and progesterone, thereby reducing their concentration by up to $50 \%$. This increases the risk of pregnancy in patients taking oral contraceptives and suggests the need to increase the dose of steroidal from 35 to $50 \mathrm{mg}$ if an enzyme inducing anti-epileptic drug is given (Wyllie, 2001).

Professionals including qualified physicians are still prescribing irrational drug combinations, banned drugs and withdrawn drugs. There is a need of mass awareness among physicians and consumers about the concept of essential medicines and use of rational combinations (Salman et al., 2008). Ethiopia is among those sub-Saharan countries affected by epilepsy chronic neurological disease. In Ethiopia the annual incidence of epilepsy is 64 in 100,000 inhabitants (Tekle-Haimanot et al., 1997).

The choice of the most appropriate drug treatment for a patient with seizures depends upon the accurate classification of the seizures, the type of epilepsy or epileptic syndromes, and drug such as availability and accessibility, efficacy, side effect profile and ease of use as well as patient factors. The aim of therapy is to minimize the recurrence of the seizures and the adverse drugs. Over $80 \%$ of epileptic patients can achieve a significant reduction in seizure frequency with one drug alone (Herks, 1994). The ultimate goal of treatment for epilepsy is no seizure and no side effects with an optimal quality of life. The best quality of life is associated with a seizure free state (Vickry et al., 1994).

Controlling seizure during pregnancy is vital, as seizures are likely to have an adverse effect on developing fetus. The most common and worrying adverse effects for pregnant women with epilepsy relate to the potential teratogenesis of AEDS. Teratogenic effects are classified as either major malformations or minor anomalies, and both have been associated with maternal AED use.

Although seizures are common in the pediatric age group and occur in approximately $10 \%$ of children but current antiepileptic drugs (AEDs) cannot control seizures in 20 to $30 \%$ of patients. Therefore, new AEDs with greater efficacy and fewer side effects are fiercely needed (Aicard, 1994). A retrospective drug use evaluation is the simplest means to be performed after a patient has received the medication. It may detect patterns in prescribing, dispensing or administering drugs to prevent recurrence of inappropriate use and serves as a means for developing prospective standards and target interventions.

\section{MATERIALS AND METHODS}

The study was conducted in Jimma university specialized teaching hospital (JUSH), in South west Ethiopia from June 25 to July 5, 2015 to assess mode of therapeutics and its outcome among epileptic patients. A facility based retrospective cross-sectional study design was employed using quantitative method of data collection. The sample size was determined by JCAHO criteria. Accordingly, $5 \%$ of the total patient medical records with AEDs prescription were drawn by simple random sampling method. A total of 154 patients' medical cards were reviewed. All incomplete and lost to follow-up patients' medical records were excluded by replacing them with the next one in numerical order from the same category patients' cards collection shelf.

Standard questionnaire containing the variables to be measured was used to collect secondary data from the selected patients' records. Three trained graduating class medical intern collected the data. Data was cleaned, checked for completeness and analyzed using SPSS version 20 for window. The results was interpreted and presented by using tables and narration. Ethical clearance was obtained from College of Health Sciences.

\section{RESULTS}

\section{Socio-demographic characteristics}

A total of 154 patients' cards were reviewed. Among these, $95(61.7 \%)$ subjects were of males. Majority of the patients, $74(48.1 \%)$, were in the age group of 5 to 18 years followed by $58(37.7 \%)$ in the age group 19 to 65 years among others. Among females participants, 54 $(91.6 \%)$ were neither pregnant nor lactating (Table 1$)$.

\section{Classification of epileptic seizures}

Generalized tonic-clonic seizures accounted for $76.3 \%$ followed by uncategorized epilepsy (18.6\%). Each of the other three seizures (generalized-tonic, idiopathic and secondary generalized) were less common and accounted for 1.2, 2.3 and 1.6\%, respectively (Table 2). 
Table 1. Socio-demographic characteristics of patients that have used AEDs in epilepsy clinic of JUSH, southwest Ethiopia, January 2015.

\begin{tabular}{|c|c|c|c|c|}
\hline \multicolumn{3}{|c|}{ Socio-demographic variable } & Antiepileptic drugs & Percentage \\
\hline \multirow{3}{*}{ Sex } & \multicolumn{2}{|c|}{ Male(M) } & 95 & 61.7 \\
\hline & \multicolumn{2}{|c|}{ Female(F) } & 59 & 38.3 \\
\hline & \multicolumn{2}{|c|}{ Total } & 154 & 100 \\
\hline \multirow{9}{*}{ Age grou } & \multirow{2}{*}{$<5$} & $M$ & 5 & 3.2 \\
\hline & & $\mathrm{F}$ & 4 & 2.6 \\
\hline & \multirow{2}{*}{$5-18$} & M & 44 & 28.6 \\
\hline & & $\mathrm{F}$ & 30 & 19.5 \\
\hline & \multirow{2}{*}{$19-65$} & $M$ & 38 & 24.6 \\
\hline & & $\mathrm{F}$ & 20 & 13 \\
\hline & \multirow{2}{*}{$>65$} & M & 8 & 5.2 \\
\hline & & $\mathrm{F}$ & 5 & 3.3 \\
\hline & \multicolumn{2}{|l|}{ Total } & 154 & 100 \\
\hline \multirow{4}{*}{ Female } & \multicolumn{2}{|c|}{ Pregnant } & 3 & 5 \\
\hline & \multicolumn{2}{|c|}{ Lactating } & 2 & 3.4 \\
\hline & \multicolumn{2}{|c|}{ Neither of the two } & 54 & 91.6 \\
\hline & \multicolumn{2}{|l|}{ Total } & 59 & 100 \\
\hline
\end{tabular}

Table 2. Classification of epileptic seizure in epilepsy clinic of JUSH southwest Ethiopia, January 2015.

\begin{tabular}{clcc}
\hline S. no & Classification of seizures & No. of patients & Percentage \\
\hline 1 & Generalized tonic-clonic seizure & 117 & 76.3 \\
2 & Uncategorized epilepsy & 30 & 18.6 \\
3 & Secondary generalized seizure & 2 & 1.2 \\
4 & Generalized tonic seizure & 3 & 2.3 \\
5 & Idiopathic seizure & 2 & 1.6 \\
& Total & 154 & 100 \\
\hline
\end{tabular}

\section{Utilization pattern of AEDs}

A total of 170 AEDs were prescribed over the study period corresponding to an average of 1.1 per patient. In this study $138(89.7 \%)$ of patients were prescribed an AED in monotherapy and $16(10.3 \%)$ with a combination of two drugs. Poly-therapy ( $\geq 3$ AEDs) was not used. In the group of patients suffering from generalized seizures, $106(68.8 \%)$ were treated with monotherapy and 16 $(10.4 \%)$ with a combination of two drugs. Phenobarbitone was the commonest monotherapy $(78.1 \%)$ followed by phenytoin (21.3\%). From the dual therapies, twelve of them were phenytoin with phenobarbitone and four were phenobarbitone with carbamazepine. Phenobarbitone $(77.1 \%)$ was the most frequently prescribed AEDs, followed by phenytoin (19.4\%) and carbamazepine $(3.5 \%)$. In the group of patients suffering from generalized seizures, only older AEDs were used when comparing the prescribing pattern of AEDs (Table 3).

\section{Indications of AEDs}

Majority of patients who were placed on AED were having GTCs with percentage of $76.3 \%$. On the second place AED was majorly presented for un-categorized epilepsy of $18.6 \%$ and it was found to be difficult to conclude either correct or incorrect indication (Table 4).

\section{Tolerability and adverse effect of AEDs}

Twenty three patients had reported adverse effects related to his/her anti-epileptic therapy. Headache was 
Table 3. AEDs utilization for treatment of epileptic seizure in epilepsy clinic of JUSH southwest Ethiopia, January 2015.

\begin{tabular}{|c|c|c|c|c|c|c|}
\hline \multirow[b]{2}{*}{ AEDs } & \multicolumn{4}{|c|}{ No. of drugs } & \multicolumn{2}{|c|}{ Dosage $(\mathrm{mg} / \mathrm{d})$} \\
\hline & $\begin{array}{c}\text { Generalized } \\
\text { seizure }\end{array}$ & $\begin{array}{l}\text { Partial } \\
\text { seizure }\end{array}$ & $\begin{array}{c}\text { Uncategorized } \\
\text { epilepsy }\end{array}$ & Total & Median & Range \\
\hline Monotherapy & 2 & 16 & 30 & 138 & & \\
\hline Dual therapy & - & 16 & - & 16 & & \\
\hline Phenobarbitone & 99 & 3 & 29 & 131 & 100 & $30-300$ \\
\hline Phenytoin & 25 & 1 & 7 & 33 & 100 & $25-200$ \\
\hline Carbamazepine & 6 & 0 & 0 & 6 & 400 & - \\
\hline Total & 130 & 4 & 36 & 170 (100\%) & - & - \\
\hline
\end{tabular}

Table 4. Reasons for using AEDs in epilepsy clinic of JUSH southwest Ethiopia, January 2015.

\begin{tabular}{llcc}
\hline AEDs & Reason for use & Frequency & Percentage \\
\hline \multirow{4}{*}{ Phenobarbitone } & Generalized tonic-clonic seizure (grand mal) & 76 & 49.7 \\
& Generalized tonic seizure & 2 & 1.3 \\
& Idiopathic seizure & 2 & 1.3 \\
& Focal seizure with 2ry generalization & 4 & 2.6 \\
& Uncategorized epilepsy & 39 & 25.3 \\
Phenytoin & Generalized tonic-clonic seizure & 11 & 7.3 \\
& Uncategorized epilepsy & 4 & 2.2 \\
Carbamazepine & Generalized tonic-clonic seizure & 16 & 10.3 \\
Total & & 154 & 100 \\
\hline
\end{tabular}

Table 5. The adverse effects faced by the patients at epilepsy clinic of JUSH southwest Ethiopia, January 2015.

\begin{tabular}{clcc}
\hline S. no. & Adverse effect & Frequency & Percentage \\
\hline 1 & Headache & 15 & 65.4 \\
2 & Irritability & 5 & 21.7 \\
3 & Hypersomnia & 1 & 4.3 \\
4 & Confusion & 1 & 4.3 \\
5 & Depressed mood & 1 & 4.3 \\
& Total & 23 & 100 \\
\hline
\end{tabular}

the commonest adverse effect faced by 15 patients, which was followed by hypersomnia (1 patient), irritability (5 patients), confusion (1 patient) and depressed mood (1 patient) (Table 5).

\section{Dose and duration of AED used}

$73.9 \%$ of AED use was containing correct dose. Out of $26.1 \%$ incorrect doses, $17.7 \%$ were under dose and $8.4 \%$ were over dose regimens. $32(21.0 \%$ of AED therapy) contained problem in duration of therapy in which $28(18.3 \%)$ are short duration and $4(2.8 \%)$ are long duration (Table 6).

\section{Contra indication and patient outcome}

There was no contraindication experience in this study. Nevertheless, of the total AEDs, $14.3 \%$ contained one or more potentially interacting drug. $119(77.3 \%)$ of the patients were seizure free while $19(12.3 \%)$ patient had less than three seizure attack and $16(10.4 \%)$ had 4 to 6 seizure attacks within the last visit.

\section{DISCUSSION}

This study indicated that $61.7 \%$ AED users were males. This largest proportionality might be an indication that 
Table 6. Dose and duration of AEDs used in epilepsy clinic of JUSH southwest Ethiopia, January 2015.

\begin{tabular}{llcc}
\hline Indicator & Variable & Frequency & Percentage \\
\hline \multirow{4}{*}{ Dose } & Correct dose & 114 & 73.9 \\
& Under dose & 27 & 17.7 \\
& Over dose & 13 & 8.4 \\
& Total & 154 & 100 \\
& & & \\
\multirow{5}{*}{ Duration } & Correct duration & 114 & 73.9 \\
& Short duration & 28 & 18.3 \\
& Long duration & 4 & 2.8 \\
& No duration & - & - \\
& Total & 154 & 100 \\
\hline
\end{tabular}

males were more prone to trauma/head injury that predisposes to the problem. In addition, some females may be left untreated due to cultural and social impact. The dominant age group of the study was 5 to 18, followed by 19 to 65 . The possible reason for the higher frequency may be due to higher possible exposure of these age groups population to most of the causes of epilepsy like trauma and toxic environmental products among others.

Generalized tonic clonic seizures (76.3\%) were the most common type of epileptic seizures encountered. This result is different from a study done on drug use evaluation of AEDs at a multispecialty tertiary care teaching hospital in India (55.2\%) (Arul Kumaran et al., 2009). The difference might be related to the difference in the contextual risk factors. Secondly $25.9 \%$ of the seizures were left uncategorized. This is most probably due to the professionals are less aware of the type of epilepsy. The key to treating epilepsy is correct diagnosis of the seizure type and, when possible, the type of epilepsy. Most patients with epilepsy respond to one of the first-line AEDs; second-line agents may be useful in patients who do not respond to one or more combinations of the first-line agents. In this study, phenobarbitone $(78 \%)$ was the first-line drug prescribed, followed by phenytoin $(21.3 \%)$ in the treatment of epileptic seizures. The selection of AEDs is increasingly more complex as new agents become available. The best AED therapy is dependent on optimal seizure control and absence of unacceptable side effects (Britton, 1996). One of the most important factors in deciding which drug to prescribe is cost of AEDs and affordability of patients (Lim et al., 1997).

It is important to maintain patients on monotherapy as compliance is better, side effects are less and there is no problem of drug-to-drug interaction. In our study, monotherapy $(89.7 \%)$ was followed by dual therapy $(10.3 \%)$. A study done in UK and Singapore showed that majority of the patients were on monotherapy $(63 \%)$ ( $\mathrm{Al}$ Khaja et al., 2006). When our result was compared with the above study, there is a variation and this indicates that there is a better use of drug in JUSH than in UK. Phenobarbitone $(78.1 \%)$ was the most frequently prescribed monotherapy, followed by phenytoin (21.3\%). In contrast, carbamazepine was the commonest AED used in monotherapy (52\%) or in combination therapy, followed by valproate and phenobarbitone in Singapore. Phenytoin with phenobarbitone was the most frequent 2drug combination in our study. Polytherapy offers no advantage over monotherapy. It increases the potential for drug-drug interactions, results in failure to evaluate the individual drugs, can increase the risk of chronic toxicity (including neurocognitive problems), may affect compliance and is associated with a higher cost of medication and necessity for TDM (Arul Kumaran et al., 2009).

Our study showed that all epileptics were managed with the conventional AEDs. Phenobarbitone was the most frequently prescribed AEDs, followed by phenytoin. Regardless of the blood level, by increasing the dose of the same AED gradually to maximum tolerated dose, seizures could be controlled in many patients. Every patient has his/her own necessary dose. For example, the dose of phenbarbitone ranged from 15 to $300 \mathrm{mg}$ even though most patients needed $100 \mathrm{mg}$ per day in our study. This study has attempted to evaluate the rational use of AEDs among patients treated in epilepsy clinic of JUSH. The rational use of drugs requires that patients receive medications appropriate to their clinical needs, in doses that meet their own individual requirements, for an adequate period of time, and at the lowest cost to them (DACA, 2007). Prescribers must adhere to the STG while prescribing drugs to patients so as to ensure treatment uniformity and promote rational drug use. The study revealed that $76.3 \%$ were correctly indicated according to Ethiopia STG and $23.7 \%$ of the indications were found to be difficult to know either correct or incorrect indications since the type of epilepsy was not identified and written on the patient card. Here, our study result shows that $76.3 \%$ of indications were in line with STG or correct indication. When compare this result with the threshold set $(95 \%)$ there is no good prescribing practice towards 
the stated disease.

As adverse effect, headache was the commonest adverse effect faced, followed by hypersonic and irritability. There was correct dosing in $73.9 \%$ of AEDs use as per the criteria set for this study. But $26.1 \%$ of the prescriptions were inappropriately dosed, of which 17.7 and $8.4 \%$ were under and over dosed regimens, respectively. The study revealed that $73.9 \%$ of the prescriptions of AED were correct dose. This was less than the set criteria (95\%). This indicates that there is no better use of drug. From the study, it was indicated there was a problem in duration of therapy in $21.3 \%$ of the prescription of which 18.3 and $2.8 \%$ were short duration and long duration, respectively. The result from a study done in Bahrain in May 2004 revealed that there is low dosing frequency $(2.6 \%)$ and excessive dosing frequency $(0.8 \%)$ were noted (Al Khaja et al., 2006). The result both in low dosing frequency and longer duration is more or less similar. From the study findings, it is evident that the result of correct duration $(79.7 \%)$ was more or less approach to the set criteria (90\%). In prescribing drugs, the prescribers should strictly avoid contra-indications, unless the benefit to the patients from using the medication out ways the risks. This study has shown that none of indications of AEDs were against contra indications. So, the result is the same with the threshold of the study $(100 \%)$. This might be due to good use of AED concerning to contra-indications.

One purpose of drug use evaluation is to identify those drugs regimens which could results in clinically significant drug-drug interactions. Drug interactions are some of the commonest causes of adverse effects. When two or more drugs are administered to patients they may act independently of each other or interactions may increase or decreases the effects of the drugs concerned and may cause unexpected toxicity. It is important to remember that interactions which modify the effects of a drug may involve non-prescription drugs, non-medical agents, social drugs and certain types of foods. Seven (14.3\%) of AED therapy, as per this study, had one or more potentially interacting drugs. Ninety two $(85.7 \%)$ of AED use was free of any interacting drugs. Drug interaction results are more or less similar to the threshold (95\%) for AED medications. This may be due to poor drug history taking by the physicians. Drugs to have correct action at correct size in desirable amount on a time, should be prescribed in a right dose and frequency. However, this study indicated that the physician or other health professionals omit (forget) this information more over prescribe wrong dose, frequency, error drug-drug interaction and drug contra indicated.

\section{Conclusions}

In summary, our study revealed that generalized seizures were the most prominent seizure encountered. Monotherapy was most frequently used in all types of epileptic seizures. The selection of the AEDs corresponds almost well with the known efficacy profile for specific epileptic seizures. Most of the criteria were almost appropriate as per the set criteria of the study. Headache was the commonest adverse effect faced by the patients. The most commonly prescribed AED was phenobarbitone, followed by phenytoin for epilepsy. Most epileptics were managed with the conventional oral AEDs. There was inappropriate usage of AEDs which comprises drug interaction, inappropriate duration of treatment and incorrect dosage regimen. There is poor classification of epilepsy. The present study has revealed better use of AEDs pertaining to indications and contraindications.

\section{RECOMMENDATIONS}

It is recommended that:

1. The Federal Ministry of Health of Ethiopia has to advise method of promoting and implementing rational utilization of drugs.

2. The classification of seizure as well as the use of drugs with potential drug interactions, dose and duration problem need urgent interventions by all responsible bodies for the service delivery in the country.

3 . The prescribers should adhere more to the guide line concerning indications, contra-indications and drug interactions.

4. The duration of therapy should be according to the STG to prevent under use and over use of AEDs.

5. Health facilities should undertake retrospective and prospective drug use evaluations, availability and accessibility of drugs and factors affecting choice of drug treatments to know drug related problems and take corrective measures.

6. Drug information centers are appropriate bodies to give any information related to drugs and be established in each health facilities to answer drug related questions of prescribers and customers.

7. The government, pharmacy schools, ministry of health and pharmacists as a whole must work towards clinical pharmacy adoption which can dramatically reduce drug and therapeutic problems and health care costs.

8. Finally, it is important for health professionals to practice good medical and drug history taking and keeping, as well as good classification of disease state.

\section{Limitation of the study}

Absence of inclusion of different methods such as anticonvulsant blood level monitoring (TDM).

\section{REFERENCES}

Aicard J, 1994. Epilepsy in children. New York, Raven Press, 2th Ed, 
pp. 391-392.

Al Khaja KAJ, Al-Ansari TM, Damanhori AHH, Sequeira RP, 2006. Rational use of antimicrobials in infants in primary care of Bahrain. $J$ Trop Pediatr, 52(6): 390-393.

Arul Kumaran KSG, Palanisamy S, Rajasekaran A, 2009. Development, implementation and assessing the effectiveness of patient information leaflets on diabetes mellitus. Int $\mathrm{J}$ Pharma Techol Res, 1(4): 1541-1547.

Britton JW, 1996. Selection of antiepileptic drugs: a practical approach. Mayo Clin Proc, 71(8):778-786.

Drug administration and control Authority (DACA), 2007. Manual for Good dispensing practices, February; pp: 5-7.

Herks GK, 1994. Antiepileptics clinical applications. Aust Prescr, 17: 912.

Joint Commission on the Accreditation of Health care Organizations, 1995. Compressive accreditation manual for hospitals. Oakbrook Terrace (IL): Joint commission on the Accreditation of Health care organizations: 1994.

Kubacka RT, 1996. A primer on drug utilization review. J Am Pharm Assoc, 36(4): 257-261.

Leppik IE, 1996. Contemporary diagnosis and management of the patient with epilepsy. 2nd Ed. Newtown, PA: Handbooks in health care.

Lim S-H, Tan EK, Chen C, 2017. Patterns of anti-epileptic drug usage in a tertiary referral hospital in Singapore. Neural $\mathrm{J}$ southeast Asia, 2: 77-87

Salman MT, Akram MF, Rahamans, Khan FA, Hassen MA, Kahan SW, 2008. Drug prescribing pattern in surgical wards of teaching hospital in Northern India. Ind J Pract Doctor, 5, 2.

Schacter SS, cramer GW, Thompson GD, chaponis RJ, Mendelson MA, Lawhorne L, 1998. An evaluation of antiepiletic drug therapy in Nursing facilities, J Am Geriatr Soc. 46: 1137-1141.

Scott RA, Lhatoo SD, Sander JWAS, 2001. The treatment of epilepsy in developing countries: Where do we go from here? WHO Bulletin 2001: 79(4): 344-351.

Sridharan R, 2002. Epidemiology of epilepsy. Curr Sci, 82(6): 664-670.

Tekle-Haimanot R, Forsgren L, Ekstedt J, 1997. Incidence of epilepsy in rural central Ethiopia. Epilepsia, 38(5): 541-546.

Vickry BG, Hays RD, Rausch R, Sutherling WW, Engel J Jr, Brook RH, 1994. Quality of life of epilepsy surgery patients as compared with out patients with hypertension, diabetes, heart disease and/or depressive symptoms. Epiletes, 35: 597-607.

Wyllie E, 2001. The treatment of epilepsy, principles and practice. 3rd Ed. Philadelphia: Lippincott Williams and Wilkins.
Citation: Atalay W, Abebe C, Salgedo WB, 2017. Antiepileptic drugs use among patients attending epilepsy clinic at Jimma university teaching and referral hospital, southwest Ethiopia: Retrospective facility based cross-sectional descriptive study. Int Res J Med Med Sci, 5(3): 37-43. 